\title{
Bessel Functions for Large Arguments
}

\author{
By M. Goldstein and R. M. Thaler
}

Calculations of Bessel Functions of real order and argument for large values of the argument can be greatly facilitated by the use of the so called phase-amplitude method [1]. In this method two auxiliary functions, the amplitude and phase functions, are defined in terms of the regular and irregular solution of Bessel's equation. These auxiliary functions have the great advantage that they are monotonic functions of the argument; moreover, for large arguments these functions are slowly varying and, hence, easily amenable to computation and interpolation.

The Bessel Functions of the third kind, so-called Hankel Functions, are defined as follows:

$$
H_{\nu}^{(1)}(x)=J_{\nu}(x)+i Y_{\nu}(x)
$$

and

$$
H_{\nu}^{(2)}(x)=J_{\nu}(x)-i Y_{\nu}(x) .
$$

These definitions suggest the alternative definition of the Bessel Function in terms of an amplitude and phase function, viz.,

$$
A_{\nu} \equiv\left|H_{\nu}^{(1)}(x)\right| \equiv\left|H_{\nu}^{(2)}(x)\right|
$$

or

$$
\begin{aligned}
& H_{\nu}{ }^{(1)}=A_{\nu} e^{+i \Phi_{\nu}} \\
& H_{\nu}{ }^{(2)}=A_{\nu} e^{-i \Phi_{\nu}}
\end{aligned}
$$

so that

$$
J_{\nu}(x)=A_{\nu} \cos \Phi_{\nu}
$$

and

$$
Y_{\nu}(x)=A, \sin \Phi_{\nu}
$$

The phase function $\Phi_{\nu}(x)$ obeys the first order differential equation

$$
\frac{d \Phi_{y}}{d x}=\frac{2}{\pi x}\left[A_{v}(x)\right]^{-2}
$$

Equation (3) represents the usual Wronskian condition. The amplitude function $A$, is seen to obey the following nonlinear second order differential equation

$$
x^{2} \frac{d^{2} A_{\nu}(x)}{d x^{2}}+x \frac{d A_{\nu}(x)}{d x}+\left(x^{2}-\nu^{2}\right) A_{\nu}(x)-\left(\begin{array}{l}
2 \\
\pi
\end{array}\right)^{2}\left[A_{\nu}(x)\right]^{-3}=0 .
$$

Equation (4) follows trivially from Bessel's differential equation and equation (3).

Received 4 June 1957. This work was performed under the auspices of the U. S. Atomic Energy Commission. 
Defining $B_{v}(t)$ as

$$
B_{\nu}(t)=\sqrt{\frac{\pi t}{2}} A_{\nu}(t),
$$

where $t \equiv 1 / x$, gives the following differential equations for $\Phi_{\nu}(t)$ and $B_{\nu}(t)$

$$
\begin{gathered}
\frac{d \Phi_{\nu}(t)}{d t}=-\left[B_{\nu}(t)\right]^{-2} \\
\frac{d^{2} B_{\nu}(t)}{d t^{2}}+\frac{1}{t^{4}}\left[1-\left(\nu^{2}-\frac{1}{4}\right) t^{2}\right] B_{\nu}(t)-\left[B_{\nu}(t)\right]^{-3}=0 .
\end{gathered}
$$

A boundary condition implied by equation (2.1) and the asymptotic behavior of $H_{\nu}(x)$ as $x \rightarrow \infty$ is

$$
\lim _{t \rightarrow 0} \frac{d^{2} B_{v}(t)}{d t^{2}}=0
$$

so that near $t=0, B_{\nu}(t)$ is approximately given by the solution of equation (7) with

This yields

$$
\frac{d^{2} B_{\nu}(t)}{d t^{2}}=0
$$

$$
B_{\nu}(t) \approx t\left[1-\left(\nu^{2}-\frac{1}{4}\right) t^{2}\right]^{-1} .
$$

Equation (9) represents the so-called JWKB approximation [2] to the amplitude function, and

$$
\Phi_{\nu}(t)=\frac{1}{t}+\int_{0}^{t}\left(\frac{1}{u^{2}}-\frac{1}{B^{2}}\right) d u-\left(\nu+\frac{1}{2}\right) \frac{\pi}{2} .
$$

The JWKB approximation for $\Phi_{\nu}(t)$ consists of the substitution of equation (9) in equation (10)

Power series expansion about $t=0$ for equations $(6,7)$ are readily obtained. One finds that

$$
\left\{B_{v}(t) / t\right\}=1+a_{2} t^{2}+a_{4} t^{4}+a_{6} t^{6}+a_{8} t^{8}+a_{10} t^{10}+\cdots
$$

where

$$
\begin{aligned}
& a_{2}=\frac{1}{4} \alpha \\
& a_{4}=\frac{5}{32} \alpha^{2}-\frac{3}{8} \alpha \\
& a_{8}=\frac{15}{128} \alpha^{8}-\frac{37}{32} \alpha^{2}+\frac{15}{8} \alpha \\
& a_{8}=\frac{195}{2048} \alpha^{4}-\frac{611}{256} \alpha^{3}+\frac{1821}{128} \alpha^{2}-\frac{315}{16} \alpha \\
& a_{10}=\frac{663}{8192} \alpha^{5}-\frac{4199}{1024} \alpha^{4}+\frac{29811}{512} \alpha^{8}-\frac{2223}{8} \alpha^{2}+\frac{2835}{8} \alpha
\end{aligned}
$$


with

$$
\alpha \equiv\left(\nu^{2}-\frac{1}{4}\right)
$$

and

(12) $t\left[\Phi_{\nu}+\left(\nu+\frac{1}{2}\right) \frac{\pi}{2}\right]=1+b_{2} t^{2}+b_{4} t^{4}+b_{6} t^{6}+b_{8} t^{8}+b_{10} t^{10}+\cdots$

where

$$
\begin{aligned}
& b_{2}=\frac{1}{2} \alpha \\
& b_{4}=\frac{\alpha^{2}}{24}-\frac{\alpha}{4} \\
& b_{6}=\frac{1}{80} \alpha^{8}-\frac{7}{20} \alpha^{2}+\frac{3}{4} \alpha \\
& b_{8}=\frac{1}{1792} \alpha^{4}-\frac{95}{224} \alpha^{3}+\frac{807}{224} \alpha^{2}-\frac{315}{56} \alpha \\
& b_{10}=\frac{7}{2304} \alpha^{5}-\frac{35}{72} \alpha^{4}+\frac{1975}{192} \alpha^{3}-58 \alpha^{2}+\frac{315}{4} \alpha .
\end{aligned}
$$

Alternatively, the amplitude function may be expressed in terms of the following series

$$
\frac{B_{r}(t)}{t}=\left[1-\alpha t^{2}\right]^{-1}\left\{1+c_{2} t^{2}+c_{4} t^{4}+c_{6} t^{6}+c_{8} t^{8}+c_{10} t^{10}+\cdots\right\}
$$

where

$$
\begin{aligned}
& c_{2}=0 \\
& c_{4}=-\frac{3}{8} \alpha \\
& c_{8}=-\frac{17}{16} \alpha^{2}+\frac{15}{8} \alpha \\
& c_{8}=-\frac{33}{16} \alpha^{3}+\frac{1761}{128} \alpha^{2}-\frac{315}{16} \alpha \\
& c_{10}=-\frac{27}{8} \alpha^{4}+\frac{6975}{128} \alpha^{3}-\frac{17469}{64} \alpha^{2}+\frac{2835}{8} \alpha .
\end{aligned}
$$

Equation (13) represents an expansion about the JWKB approximation. The series in Equation (13) is semi-convergent. Substitution of Equation (13) into Equation (6) may be used to obtain an explicit "JWKB" series for $\Phi_{v}(t)$. However, the analytic form of the coefficients is so complicated as to be of little practical value. 
The amplitude and phase functions for $\nu=0, \frac{1}{3}, \frac{1}{2}, \frac{2}{3}, 1$ are plotted in figures 1 and 2 respectively. In figure 1 the function

$$
\left[\frac{B_{v}(t)}{t}\right]
$$

is plotted against $t$. In figure 2 the function

$$
\left[t\left\{\Phi_{\nu}(t)+\left(\nu+\frac{1}{2}\right) \frac{\pi}{2}\right\}\right]
$$

is plotted against $t$. In terms of these functions the Bessel Functions $J_{\nu}(x)$ and $Y_{\nu}(x)$ become

$$
\begin{aligned}
& J_{\nu}(x)=\left[\frac{B_{\nu}(t)}{t}\right] \sqrt{\frac{2 t}{\pi}} \cos \Phi_{\nu}(t), \\
& Y_{\nu}(x)=\left[\frac{B_{\nu}(t)}{t}\right] \sqrt{\frac{2 t}{\pi}} \sin \Phi_{\nu}(t),
\end{aligned}
$$

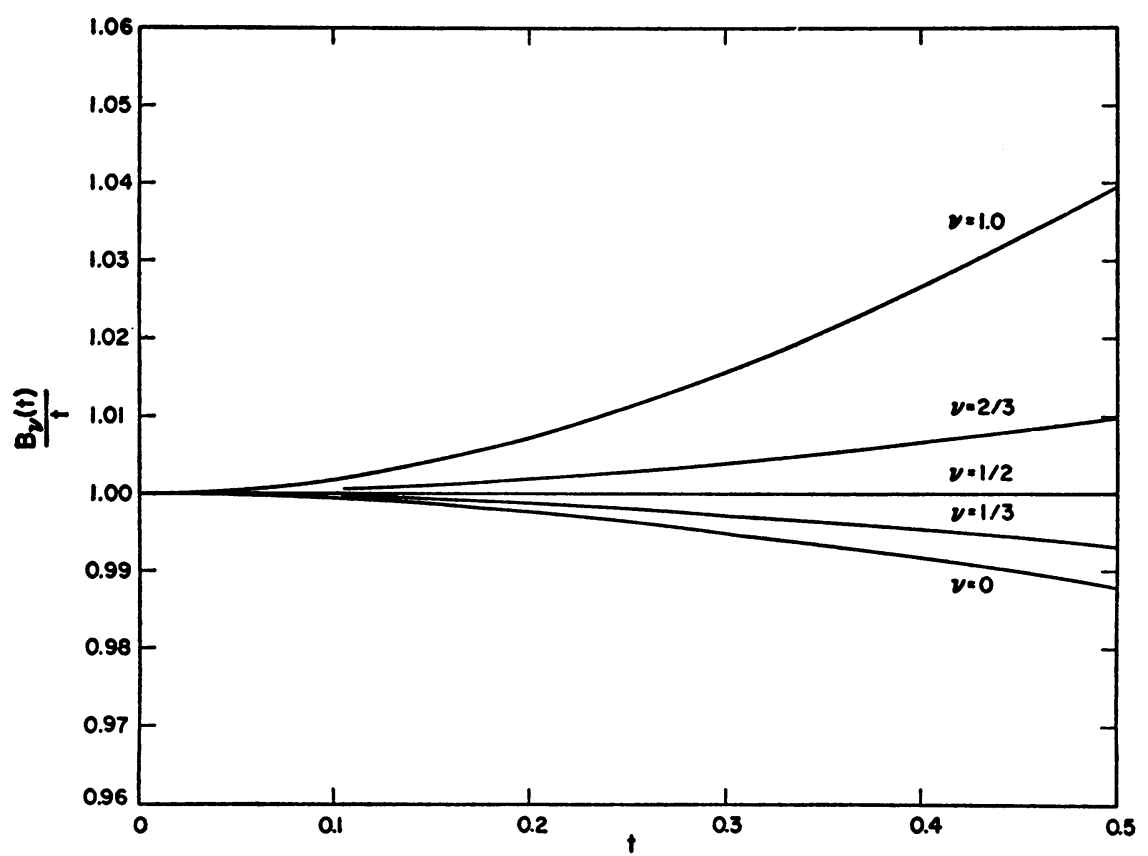

FrgURE 1. The amplitude function, $\frac{B_{v}(t)}{t}$ vs. $t$ for several values of, between zero and one. The amplitude and phase functions are defined by the relations

where

$$
\begin{aligned}
& J_{\nu}(x)=\left[\frac{B_{\nu}(t)}{t}\right] \sqrt{\frac{2 t}{2 t}} \cos \Phi_{\nu}(t) \\
& Y_{\nu}(x)=\left[\frac{B_{\nu}(t)}{t}\right] \sqrt{\frac{2 t}{2}} \sin \Phi_{\nu}(t),
\end{aligned}
$$

$$
t=\frac{1}{x}
$$


with

$$
t \equiv \frac{1}{x}
$$

For $|\nu| \leqq 1$, the series equation (11 or 13) and equation (12) yield eight figure accuracy in $J_{\nu}(1 / t)$ and $Y_{\nu}(1 / t)$ for $t \leqq \frac{1}{8}$, for the number of terms shown. This is in contrast with the usual asymptotic expansions which yield comparable accuracy only for $t \leqq 1 / 20$, and which converge much more slowly in the region of good convergence.

For $\nu=\frac{1}{2}, \alpha=0$, so that the coefficients in equations (11) and (12) vanish. Therefore, for $\nu=\frac{1}{2}$ the series reduce to single terms, yielding

$$
\begin{aligned}
& J_{\frac{1}{3}}(x)=\sqrt{\frac{2}{\pi x}} \sin x \\
& Y_{\frac{1}{3}}(x)=-\sqrt{\frac{2}{\pi x}} \cos x
\end{aligned}
$$

or

$$
\begin{aligned}
& A_{\frac{1}{1}}(x)=\sqrt{\frac{2}{\pi x}} \\
& \Phi_{\frac{1}{3}}(x)=x-\frac{\pi}{2} .
\end{aligned}
$$

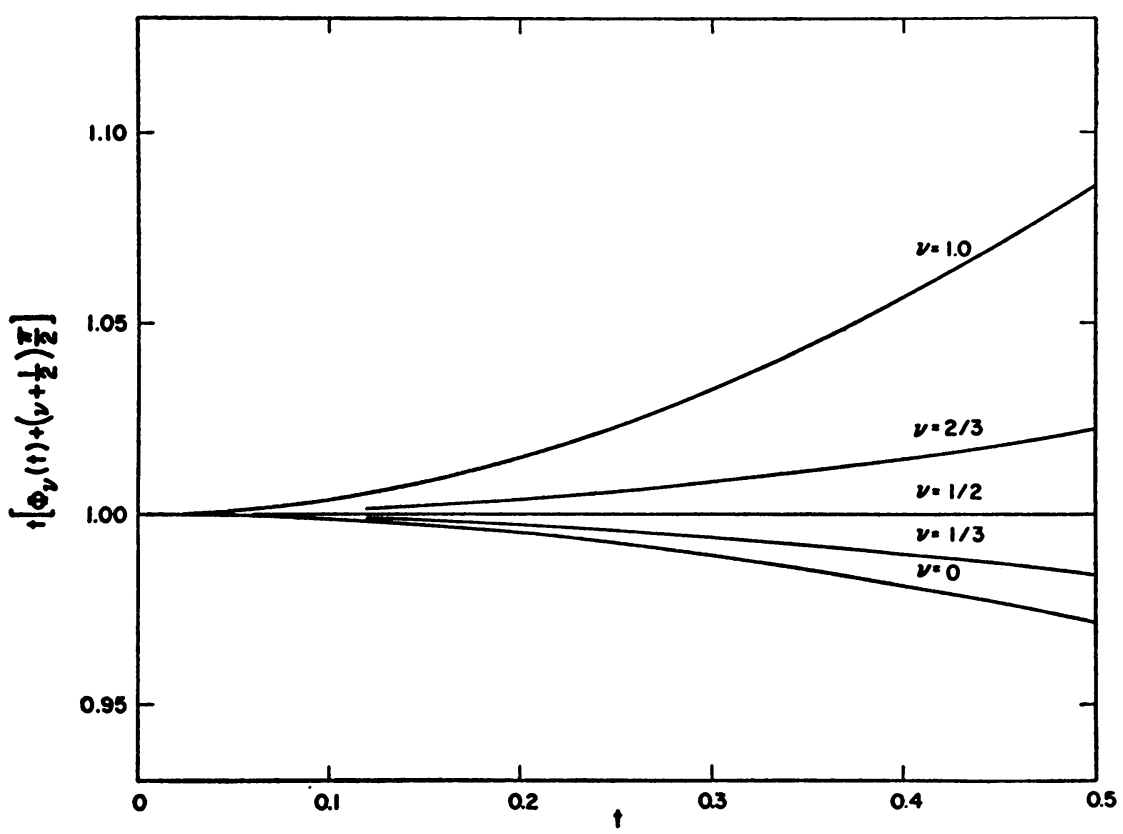

FIGURE 2. The phase function $t\left[\Phi_{\nu}(t)+\left(\nu+\frac{1}{2}\right) \frac{\pi}{2}\right]$ vs. $t$, for several values of $\nu$ between zero and one. 
It is also clear that the series are most rapidly convergent for $\nu$ close to $\frac{1}{2}$.

For computational purposes it is evident that greatest accuracy will result through the use of equations (11) and (12) for $|\nu| \leqq 1$. The appropriate recurrence techniques may be used to obtain numerical values for $|\nu|>1$.

Equations (11) and (12) may be regarded as starting series for the differential equations equations (6) and (7). For $|\nu| \leqq 1$ equations (6) and (7) may be integrated numerically starting at $t \sim \frac{1}{8}$ and give good accuracy to $t \sim \frac{1}{2}$. Thus the method described above can be used to generate values of $J_{\nu}(x)$ and $Y_{\nu}(x)$ for $x \geqq 2$.

The Bessel Functions of imaginary argument $I_{\nu}(x)$ and $K_{\nu}(x)$ are amenable to similar treatment. The phase-amplitude functions are defined as

$$
\begin{aligned}
& I_{\nu}=\sqrt{\frac{2}{\pi x}} C_{\nu} \sinh \phi_{\nu} \\
& K_{\nu}=\sqrt{\frac{\pi}{2 x}} C_{\nu} e^{-\phi_{\nu} .}
\end{aligned}
$$

These definitions are chosen so that

$$
C_{\frac{1}{3}}=1
$$

and

or

$$
\phi_{1}=x
$$

$$
\begin{aligned}
& I_{\frac{1}{3}}(x)=\sqrt{\frac{2}{\pi x}} \sinh x \\
& K_{\frac{1}{3}(x)}=\sqrt{\frac{\pi}{2 x}} e^{-x} .
\end{aligned}
$$

The phase function $\phi$, obeys the first order differential equation

$$
\frac{d \phi_{\nu}(t)}{d t}=-\left[D_{v}(t)\right]^{-2}
$$

and the amplitude function $D_{\nu}=t C_{\nu}$, obeys the nonlinear second order differential equation

$$
-\frac{d^{2} D_{\nu}(t)}{d t^{2}}+\frac{1}{t^{4}}\left[1+\left(\nu^{2}-\frac{1}{4}\right) t^{2}\right] D_{\nu}(t)-\left[D_{\nu}(t)\right]^{-3}=0
$$

The series expansions about $t=0$ are

$$
\left\{\frac{D_{\nu}(t)}{t}\right\}=1-a_{2} t^{2}+a_{4} t^{4}-a_{6} t^{6}+a_{8} t^{8}-a_{10} t^{10}+\cdots,
$$

and

$$
t \phi_{v}(t)=1-b_{2} t^{2}+b_{4} t^{4}-b_{6} t^{6}+b_{8} t^{8}-b_{10} t^{10}+\cdots .
$$

Alternatively, in the JWKB type approximation, the amplitude function is given by the series

$$
\frac{D_{v}(t)}{t}=\left[1+\alpha t^{2}\right]^{-1}\left\{1-c_{2} t^{2}+c_{4} t^{4}-c_{6} t^{6}+c_{8} t^{8}-c_{10} t^{10}+\cdots\right\},
$$


where the $a_{\mu}, b_{\mu}$ and $c_{\mu}$ are defined as in equations (11), (12), and (13) and

$$
\phi_{\nu}(t)=\frac{1}{t}+\int_{0}^{t}\left(\frac{1}{u^{2}}-\frac{1}{D^{2}}\right) d u .
$$

The amplitude and phase functions for $\nu=0, \frac{1}{3}, \frac{1}{2}, \frac{3}{3}, 1$ are plotted in figures 3 and 4 respectively. In figure 3 the function

$$
\left[D_{\nu}(t) / t\right]
$$

is plotted against $t$. In figure 4 the function

$$
\left[t \phi_{\nu}(t)\right]
$$

is plotted against $t$.

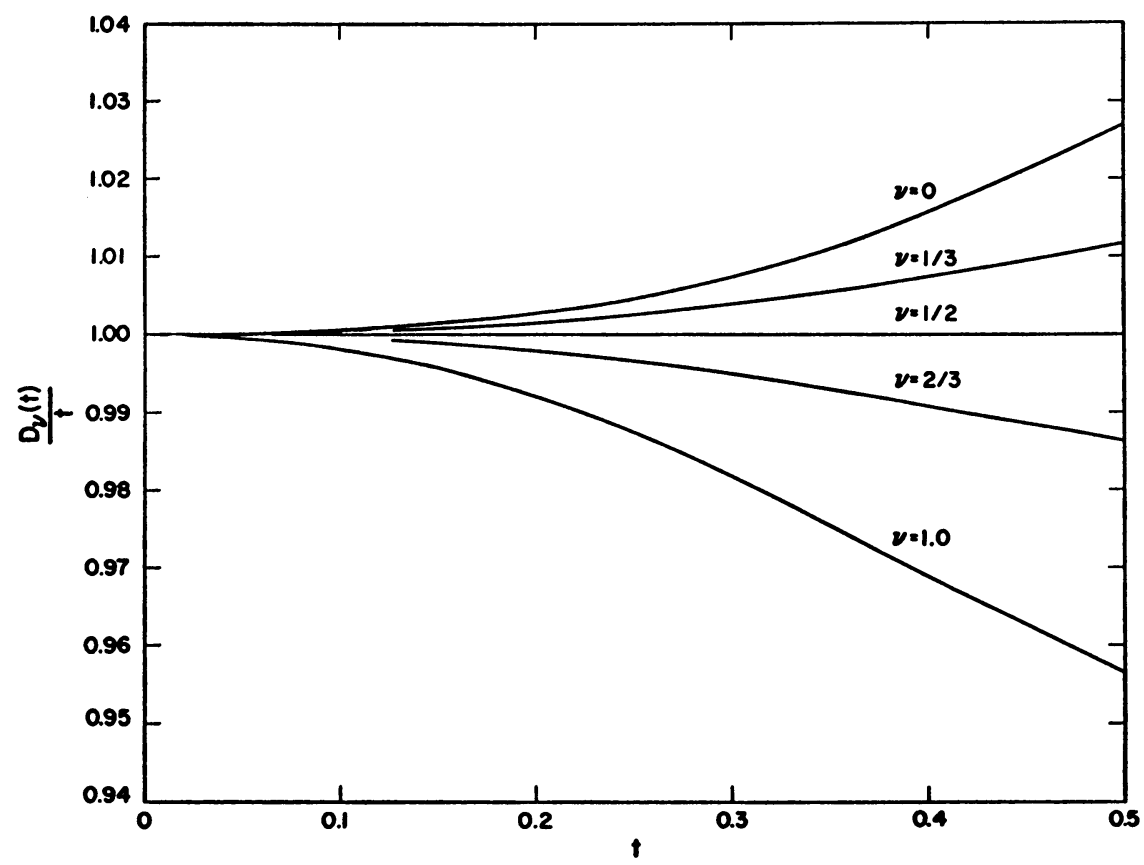

FIGURE 3. The amplitude function $\frac{D_{\nu}(t)}{t}$ vs. $t$ for several values of $\nu$ between zero and one. The amplitude and phase functions are defined by the relations

where

$$
\begin{aligned}
& I_{\nu}(x)=\left[\frac{D_{\nu}(t)}{t}\right] \sqrt{\frac{2 t}{\pi}} \sinh \phi_{\nu}(t) \\
& K_{v}(x)=\left[\frac{D_{\nu}(t)}{t}\right] \sqrt{\frac{2 t}{\pi}} \frac{\pi}{2} \exp \left[-\phi_{\nu}(t)\right]
\end{aligned}
$$

$$
t=\frac{1}{x}
$$




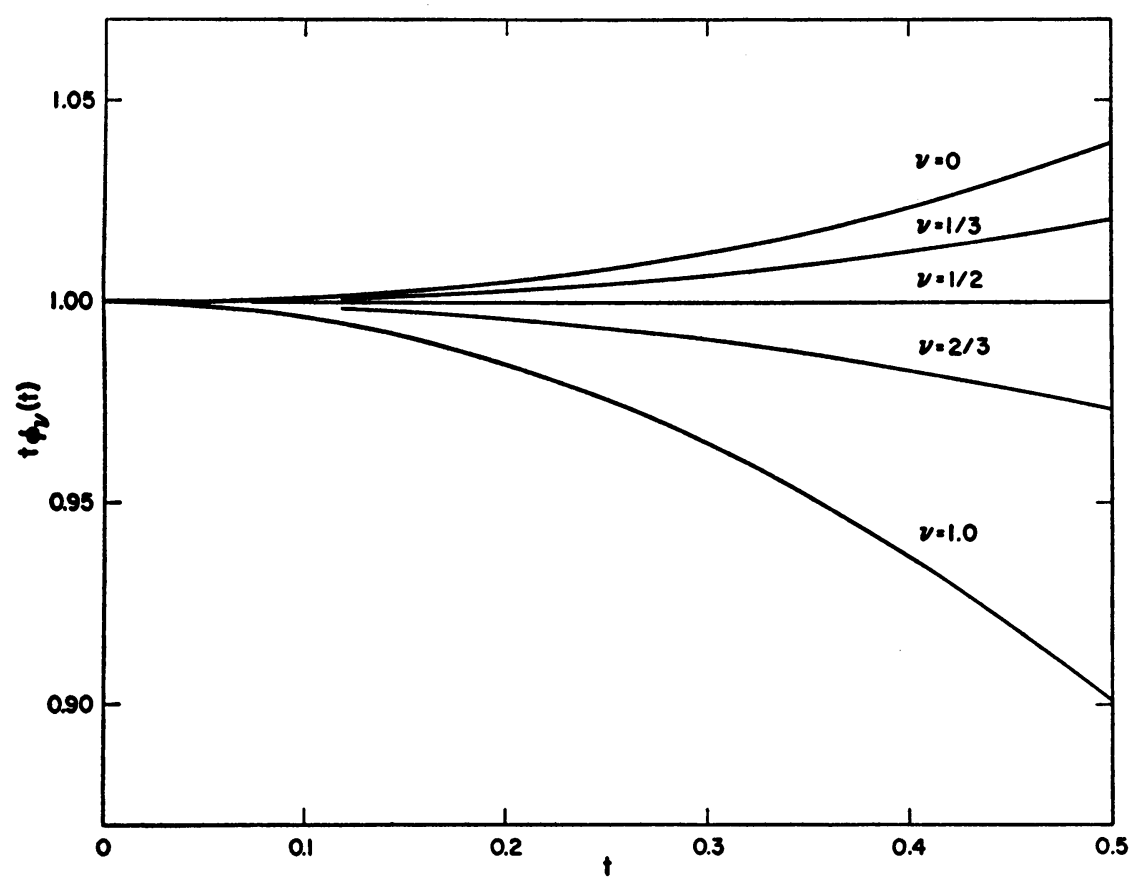

FIgURE 4. The phase function $t \phi_{y}(t)$ vs. $t$, for several values of $\nu$ between zero and one.

In terms of these functions the Bessel Functions of imaginary argument $I_{\nu}(x)$ and $K_{p}(x)$ become

with

$$
\begin{aligned}
& I_{\nu}(x)=\left[\frac{D_{\nu}(t)}{t}\right] \sqrt{\frac{2 t}{\pi}} \sinh \phi_{v}(t) \\
& K_{\nu}(x)=\left[\frac{D_{\nu}(t)}{t}\right] \sqrt{\frac{2 t}{\pi}} \frac{\pi}{2} e^{-\phi_{\nu}(t)}
\end{aligned}
$$

$$
t \equiv \frac{1}{x}
$$

The series, equations (19) and (20), can provide starting values for the numerical integration of the differential equations, equations (17) and (18). These series give seven figure accuracy for $|\nu| \leqq 1$ for $x \geqq 8$, for the number of terms shown. However, while straightforward numerical integration of equations (17) and (18) yields accurate values for $K_{v}(x)$ for $x \geqq 2$, the values for $I_{v}(x)$ rapidly deteriorate. The reason for this is clear. At $x=8$, e.g., the starting values are accurate to eight figures. The functions $\tilde{I}_{v}(x)$ and $\tilde{K}_{v}(x)$ so calculated may then be written as

$$
\begin{aligned}
I_{\nu}(x) & =I_{\nu}(x)+\rho K_{\nu}(x) \\
\tilde{K}_{\nu}(x) & =K_{\nu}(x)+\sigma I_{\nu}(x),
\end{aligned}
$$

where $\rho K_{\nu}(x)$ and $\sigma I_{\nu}(x)$ represent the truncation error, and $\rho$ and $\sigma$ are constants. 
As $x$ decreases, $K_{\nu}(x)$ increases rapidly while $I_{\nu}(x)$ decreases rapidly. Therefore, the error introduced by the truncation, decreases in the case of $K_{\nu}(x)$, and increases in the case of $I_{\nu}(x)$. This difficulty is avoided by calculating $\rho$. That is, at $x=2$, e.g., where $K_{\nu}(x)$ is still accurately given by the solution of the differential equations, equations (17) and (18)

$$
\rho=\frac{\tilde{I}_{\nu}(x)-I_{\nu}(x)}{K_{\nu}(x)} .
$$

At $x=2, I_{\nu}(x)$ is easily obtained by standard methods, e.g. the defining series, or integral representation.

M. Goldstein

Los Alamos Scientific Laboratory,

R. M. Thaler

Los Alamos, New Mexico

1. W. E. Milne, "The numerical determination of characteristic numbers," Phys. Rev., v. 35,1930 , p. $863-867$.

2. HAROLD JEFFREYS, "On certain approximate solutions of linear differential equations of the second order," London Math. Soc., Proc., v. 23, 1923, p. 428-436.

The mathematical method first given by Jeffreys was independently derived in connection with an important physical problem by

a. G. WentzeL, "Eine Verallgemeinerung der Quantenbedingungen für die Zwecke der Wellenmechanik," Zeits. f. Physik, v. 38, 1926, p. 518-529.

b. H. A. Kramers, "Wellenmechanik und halbzahlige Quantisierung," Zeits. f. Physik, v. 39,1926, p. 828-840.

c. L. Brillouin, "La mécanique ondulatoire de Schrödinger; une méthode générale de résolution par approximations sucessives," Comptes Rendus, v. 183, 1926, p. 24-26.

d. L. Brillouin, "Remarques sur la mécanique ondulatoire," J. de Physique, v. 7, 1926, p. $353-368$, and is commonly referred to as the JWKB approximation.

\title{
Numerical Experiments in Potential Theory Using the Nehari Estimates
}

\author{
By U. W. Hochstrasser
}

1. Introduction. It is well known that the Dirichlet problem can be solved by using a set of harmonic functions which are orthogonal over the boundary [7]. Prior to the availability of high speed computers, however, the method was not frequently used since generating sets of orthogonal functions by hand computations is very laborious. With the advent of high speed computers interest in the method has been renewed and several papers have appeared recently $[1,2,3]$.

In using the method, the numerical analyst is particularly interested in estimates of the error committed by replacing infinite orthogonal expansions by finite ones. Nehari [1] recently has given such estimates for a number of Dirichlet problems. The purpose of the present paper is to report the results of some numerical experiments which were designed to investigate how close these estimates come to the actual errors in certain particular examples. An existing computer program [4] was used for the orthogonalization of a given set of vectors on the SEAC com-

Received 27 March 1957. The preparation of this paper was sponsored by the Office of Scientific Research of the Air Research and Development Command, United States Air Force, while the author was a guest worker at the National Bureau of Standards. 